\title{
APPROXIMATION BY THE TRANSLATES OF A SINGLE FUNCTION
}

\section{FRANK QUIGLEY}

In 1941 Seidel and Walsh [3] proved the existence of an entire function $F$ of a complex variable such that every function analytic in a simply connected region of the complex plane is the uniform limit on compact sets of a sequence of translates of $F$. This result generalizes a theorem of G. D. Birkhoff [1] on entire functions. In the present note an analogous theorem is proved for continuous real or complex functions on a more general class of topological spaces where the role of polynomial approximation in the above proofs is assumed by a sequence of functions constructed using Urysohn's lemma.

Let $X$ be a locally compact hausdorff space with the following properties: there exist countable sequences $\left\{C_{n}\right\}$ and $\left\{\sigma_{n}\right\}$ of disjoint compact sets and homeomorphisms of $X$ onto itself, respectively, such that for every compact $K$, Ia. $K \cap C_{n}=\varnothing$ and Ib. $K \subset C_{n} \sigma_{n},{ }^{1}$ except for finitely many $n$. Such an $X$ is evidently not compact but is countable at infinity, since each point lies in some $C_{n} \sigma_{n}$. Thus the compact open topology on the algebra $\mathfrak{A}$ of all continuous real or complex valued functions on $X$ is the topology of sequential convergence in a suitable Fréchet metric on $\mathfrak{A}$.

THEOREM. Let $X$ be a locally compact hausdorff space with properties $\mathrm{Ia}$ and $\mathrm{Ib}$, and let $\mathfrak{Y}$ be a countable family of continuous real or complex functions on $X$. Then there exists a continuous real or complex function $F$ on $X$ such that every uniform limit on compact sets of functions in $\mathfrak{Y}$ is the limit of a sequence of the functions $F \circ \sigma_{n}^{-1}$.

First we find an infinite subsequence $\left\{C_{m}\right\}$ of $\left\{C_{n}\right\}$ and sets $\Delta_{m}$ and $W_{m}$, compact and open respectively, such that $\Delta_{m} \subset W_{m} \subset C_{m}$ and such that $\left\{\Delta_{m} \sigma_{m}\right\}$ retains property Ib. Since $X$ is locally compact, the interiors $U_{n}$ of $C_{n} \sigma_{n}$ are nonempty for an infinite set $J$ of integers, and $\left\{U_{j}, j \in J\right\}$ has property Ib. In fact, each compact $K$ has a compact neighborhood $N$, and $N \subset C_{j} \sigma_{j}$ for all $j$ large; thus the interior of $N$, which contains $K$, is contained in $U_{j}$. The $C_{j} \sigma_{j}, j \in J$, are compact, so that for each $j$ there is a least integer $\gamma(j) \in J$ such that $C_{j} \sigma_{j} \subset U_{\gamma(j)}$. For each $m$ in the range $\mathrm{M}$ of the function $\gamma$ choose $j$ such that $\gamma(j)$ $=m$ and define $\Delta_{m}=\bar{U}_{j} \sigma_{m}^{-1}$ and $W_{m}=U_{m} \sigma_{m}^{-1}$. If $j$ is not in the range

Received by the editors March 5, 1957.

1 We write $C_{n} \sigma_{n}$ instead of $\sigma_{n}\left(C_{n}\right)$. All indices are positive integers. 
of $\gamma$, then $C_{j} \sigma_{j} \subset U_{\gamma(j)}$, and $\left\{C_{m} \sigma_{m}, m \in \mathrm{M}\right\}$ retains property Ib. Thus $\left\{\Delta_{m} \sigma_{m}\right\}$ has property Ib also. Evidently $\Delta_{m} \subset W_{m} \subset C_{m}$, since $\bar{U}_{j} \subset U_{m}$ $\subset C_{m} \sigma_{m}$. Reindex the $\Delta$ 's, $W$ 's, and $C$ 's, using all the positive integers.

Next we construct compact sets $\Gamma_{m}$ with the properties IIa. $\Gamma_{m} \subset \Gamma_{m+1} ; U \Gamma_{m}=X$; every compact $K \subset \Gamma_{m}$, if $m$ is large. And IIb. $\bigcup_{i=1}^{m} \Delta_{i} \subset \Gamma_{m}$ and $\Gamma_{m} \cap \Delta_{m+1}=\varnothing$. Define $\Gamma_{m}$ as

$$
\bigcup_{i=1}^{m} \bar{W}_{i} \cup\left[\bar{U}_{m} \cap C\left(\bigcup_{m+1}^{\infty} W_{i}\right)\right] \text {. }
$$

Now $\bar{U}_{m} \subset \bar{U}_{m+1}$, and $\mathbf{C}\left(\cup_{m+1}^{\infty} W_{i}\right) \subset \mathbf{C}\left(\cup_{m+2}^{\infty} W_{i}\right)$; thus $\Gamma_{m} \subset \Gamma_{m+1}$. Since $\bar{U}_{m}$ is compact, so is $\Gamma_{m}$. Further

$$
\bar{U}_{m} \subset\left[\bar{U}_{m} \cap C\left(\bigcup_{m+1}^{\infty} W_{i}\right)\right] \cup \bigcup_{1}^{\infty} W_{i} \subset \bigcup_{1}^{\infty} \Gamma_{m} \text {, and } U \Gamma_{m}=X .
$$

Since $W_{m} \subset C_{m}$, each compact $K$ meets only finitely many $W_{m}$ and lies in all but finitely many $\bar{U}_{m}$; thus $K \subset \Gamma_{m}$ for all $m$ large. The first part of IIb is trivial. For the second part, observe that $\bar{W}_{m} \subset C_{m}$ and $C_{m} \cap \Delta_{m+1}=\varnothing$. Also $\left[\bar{U}_{m} \cap \mathbf{C}\left(\mathrm{U}_{m+1} W_{i}\right)\right] \subset \mathbf{C} W_{m+1}$ and $\Delta_{m+1} \subset W_{m+1}$. Thus $\Gamma_{m} \cap \Delta_{m+1}=\varnothing$.

We are now in a position to construct $F$. Let $\left\{f_{m}\right\}$ be the family $\mathfrak{V}$ indexed by the positive integers, in such a way that each function is repeated countably often, and construct continuous functions $\alpha_{m}$, $\beta_{m}$, and $g_{m}$ as follows, using Urysohn's lemma:

$$
\begin{gathered}
\alpha_{m}(x)=\left\{\begin{array}{ll}
0 \text { on } \Delta_{m}, \\
1 \text { on } \Gamma_{m-1},
\end{array} \quad \beta_{m}(x)=\left\{\begin{array}{lll}
1 & \text { on } \Delta_{m}, \\
0 & \text { on } \Gamma_{m-1},
\end{array}\right.\right. \\
\left\{\begin{array}{l}
g_{1}(x)=f_{1}(x), \\
g_{m}(x)=\alpha_{m}(x) g_{m-1}(x)+\beta_{m}(x) f_{m}\left(x \sigma_{m}\right) .
\end{array}\right.
\end{gathered}
$$

Observe that $g_{m}(x)=g_{m-1}(x)$ on $\Gamma_{m-1}$ and that $g_{m}(x)=f_{m}\left(x \sigma_{m}\right)$ on $\Delta_{m}$. Since each compact $K$ lies in all $\Gamma_{m}$ from some $m$ on, the sequence $\left\{g_{m}\right\}$ converges uniformly on compact sets to a limit $F$; this function is continuous, since it coincides with a continuous function on each compact set, and the space $X$ is locally compact. Now $F(x)=g_{m}(x)$ on $\Gamma_{m} \supset \Delta_{m}$, so that $F(x)=f_{m}\left(x \sigma_{m}\right)$ on $\Delta_{m}$. Let $y \in \Delta_{m} \sigma_{m}$ and write $y=x \sigma_{m}$ for some $x \in \Delta_{m}$. Then $F\left(y \sigma_{m}^{-1}\right)=F(x)=f_{m}\left(x \sigma_{m}\right)=f_{m}(y)$ for $y \in \Delta_{m} \sigma_{m}$. The sequence $\left\{\Delta_{m} \sigma_{m}\right\}$ has property Ib; so suppose that the sequence $\left\{f_{n_{i}}\right\}$ of functions from $\mathfrak{Y}$ converges uniformly on compact sets to a function $f$. For each compact $K$ there is an $i_{0}$ such that $K \subset \Delta_{n_{i}} \sigma_{n_{i}}$ 
for $i \geqq i_{0 .}{ }^{2}$ But $f_{n_{i}}(y)=F\left(y \sigma_{n_{i}}^{-1}\right)$ for $y \in \Delta_{n_{i}} \sigma_{n_{i}}, i \geqq i_{0}$. Thus $\left\{F\left(y \sigma_{n_{i}}^{-1}\right)\right\}$ converges uniformly to $f$ on $K$.

COROLlaRy 1. The theorem can be proved under hypothesis Ib and the following condition: Ia'. There exist open sets $V_{n} \supset C_{n}$ such that $V_{n} \cap C_{m}=\varnothing$, if $n \neq m$, and the set $\cup C_{n}$ is closed.

It is enough to show that for every compact $K, K \cap C_{n}=\varnothing$, except for finitely many $n$. Assume that $K \cap C_{m} \neq \varnothing$ for an infinite subset $\mathrm{M}$ of $\{n\}$, and choose $p_{m} \in K \cap C_{m}$ for each $m \in \mathrm{M}$. Then $\left\{p_{m}\right\}$ is an infinite point set, which must have a limit point $p$ in $K \cap \mathrm{cl}\left(U C_{m}\right)$. But if $p \in \operatorname{cl}\left(\cup C_{m}\right)$, then $p \in \cup C_{n}$, since $\cup C_{n}$ is closed, and so $p \in C_{r}$ for some $r$. Thus infinitely many $p_{m}$ lie in $V_{r}$, which is impossible for $m \neq r$; consequently $K \cap C_{n}=\varnothing$, for all $n$ large.

Corollary 2. If $X$ is a differentiable manifold of class $r, 1 \leqq r \leqq \infty$, and if the $\sigma_{n}$ and the $f_{n}$ are of class $r$, then $F$ can be found also of class $r$.

The functions $\alpha_{m}$ and $\beta_{m}$ can be chosen of class $r,{ }^{3}$ so that the $g_{n}$ are also of class $r$. For each point $p \in X$, choose a compact neighborhood $N$. Then for some $m, N \subset \Gamma_{m}$; on $\Gamma_{m}$ the function $F$ equals $g_{m}$, which is of class $r$. Thus $F$ has class $r$.

\section{REFERENCES}

1. G. D. Birkhoff, Démonstration d'un théorème élêmentaire sur les fonctions entières, Collected Papers, vol. III, pp. 307-309.

2. G. de Rham, Variétés différentiables, Paris, 1955.

3. W. Seidel and J. L. Walsh, On approximation by euclidean and non-euclidean translations of an analytic function, Bull. Amer. Math. Soc. vol. 47 (1941) pp. 916920.

YALE UNIVERSITY

${ }^{2}$ If $n_{i}=m_{0}$ for infinitely many $i$, then $\lim f_{n_{i}}=f_{m_{0}}$. Since $f_{m_{0}}$ occurs countably often among the $f_{m}$, it is the limit of translates of $F$.

${ }^{3}$ See for example $[2$, p. 6]. 